\title{
Detailed validation of the bidirectional effect in various Case 1 waters for application to ocean color imagery
}

\author{
K. J. Voss ${ }^{1}$, A. Morel ${ }^{2}$, and D. Antoine ${ }^{2}$ \\ ${ }^{1}$ Department of Physics, University of Miami, Coral Gables, Florida, USA \\ ${ }^{2}$ Laboratoire d'Océanographie de Villefranche, Université Pierre et Marie Curie et Centre National de la Recherche \\ Scientifique, Villefranche-sur-mer, France
}

Received: 7 June 2007 - Published in Biogeosciences Discuss.: 27 June 2007

Revised: 17 September 2007 - Accepted: 17 September 2007 - Published: 26 September 2007

\begin{abstract}
The radiance viewed from the ocean depends on the illumination and viewing geometry along with the water properties, and this variation is called the bidirectional effect. This bidirectional effect depends on the inherent optical properties of the water, including the volume scattering function, and is important when comparing data from different satellite sensors. The current model of $f / Q$, which contains the bidirectional effect, by Morel et al. (2002) depends on modeled, not measured, water parameters, thus must be carefully validated. In this paper we combined upwelling radiance distribution data from several cruises, in varied water types and with a wide range of solar zenith angles. We compared modeled and measured $L_{\text {view }} / L_{\text {nadir }}$ and found that the average difference between the model and data was less than 0.01 , while the RMS difference between the model and data was on the order of 0.02-0.03. This is well within the statistical noise of the data, which was on the order of $0.04-0.05$, due to environmental noise sources such as wave focusing.
\end{abstract}

\section{Introduction}

The upwelling radiance distributions, either beneath the interface or emerging from the water, are not isotropic, but vary with illumination and viewing conditions and also with water optical properties. Knowing how to predict this angular variation is important in satellite oceanography, as the analysis of satellite derived upwelling radiances must take into account these variations. This is particularly important when comparing different ocean color sensors as these sensors will view the same spot at different times (hence varied illumination geometry) and under different view angles. One must have a model of this variation of the radiance distribution that is dependent on a small set of parameters, but which can accu-

Correspondence to: K. J. Voss

(voss@physics.miami.edu) rately predict the variation. As the available model for Case I waters (Morel et al., 2002) is based on radiation transport computations, it is essentially theoretical in nature. Moreover, it includes assumptions and parameterizations of the inherent optical properties, as a function of the chlorophyll concentration, which presently are not fully verified (this is particularly true for the volume scattering function). Therefore a comparison between field data and model predictions over a wide range of experimental conditions is necessary. This is the objective of the present paper which makes use of recent and numerous observations in the Pacific Ocean and in the Mediterranean Sea.

The shape of the upwelling radiance distribution, $L_{u}\left(\theta_{0}, \theta_{v}, \phi\right)$, can be described by the bidirectional function, denoted $Q\left(\theta_{o}, \theta_{v}, \phi_{v}\right)$, which is defined as:

$Q\left(\theta_{o}, \theta_{v}, \phi_{v}\right)=E_{u} / L_{u}\left(\theta_{o}, \theta_{v}, \phi_{v}\right)$

where $\theta_{o}$ is the solar zenith angle, $\theta_{v}$ is the view nadir angle, $\phi$ is the azimuth between these two directions, and $E_{u}$ is the planar upward irradiance, i.e., the integral of the upward radiance field over the half space ( $2 \pi \mathrm{sr})$. The Morel et al. (2002) model, which is commonly used in satellite oceanography, characterizes the variations in $Q$ as a function of the three angles and of the chlorophyll concentration, [Chl]. [Chl] is a convenient index for Case 1 waters as it characterizes the bio-optical state of the water bodies. This model incorporates both a correction for this varying $Q$ factor, and also a correction for the $f$ factor. The $f$ factor relates the irradiance reflectance to the absorption coefficient and the backscattering coefficient, $a$ and $b_{b}$, respectively, through

$R(\lambda)=f(\lambda)\left[b_{b}(\lambda) / a(\lambda)\right]$.

where $f(\lambda)$ for a given water is also dependent on the solar zenith angle.

This paper will describe our results in validating the $Q$ factor. The problem of validating $f$ could be addressed with irradiance reflectance measurements combined with $b_{b}$ and

Published by Copernicus Publications on behalf of the European Geosciences Union. 


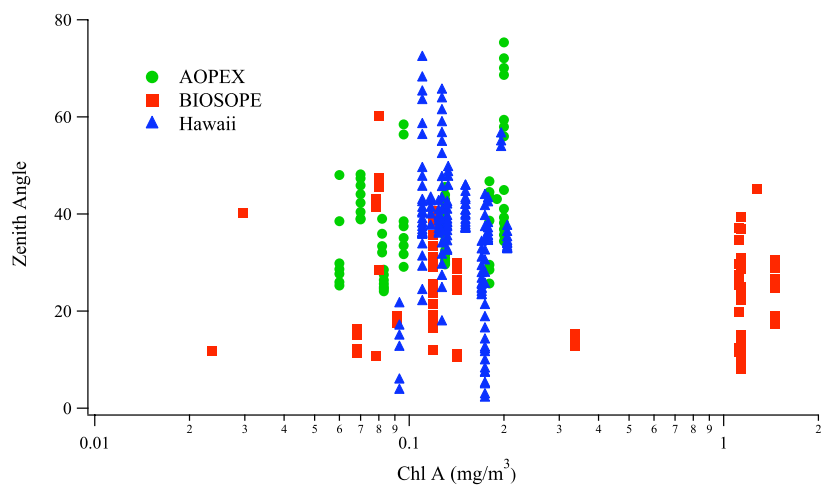

Fig. 1. Data distribution in terms of [Chl] and solar zenith angle.

$a$ measurements, but we do not have a large database of contemporaneous $b_{b}$ and $a$ coefficients for such a validation.

In the past we have had two investigations looking at the accuracy of the series of $Q$ values as predicted by models (Morel et al., 1995; Voss and Morel, 2005). Each of these was done with a different version of our radiance distribution camera systems (RADS: Voss, 1989; RADS-II, Voss and Chapin, 1992). These early instruments were large and slow and provided a limited data set. Recently we have developed a new generation of radiance distribution camera systems specifically aimed at looking at the upwelling radiance distribution (NuRADS: Voss and Chapin, 2005). With this new instrument we have an extensive set of upwelling radiance distribution data in both Case I and Case II waters. This paper will concentrate on the Case I waters, as this is where the 2002- model was designed to work. The Case II situation is much more complicated, less predictable (e.g. Loisel and Morel, 2001), and will be separately addressed in later work.

The first investigation (1995) was carried out off of California with a stable [Chl] value $\left(0.3 \mathrm{mg} \mathrm{m}^{-3}\right)$, and widely varying sun zenith angle $\left(32^{\circ}\right.$ to $\left.80^{\circ}\right)$. In contrast, the second investigation (2005), with measurements around the Baja Peninsula, encompassed a large range of chlorophyll concentrations ([Chl] from 0.14 to $11 \mathrm{mg} \mathrm{m}^{-3}$ ), whereas the sun angle remained in a rather restricted range $\left(28^{\circ}\right.$ to $\left.40^{\circ}\right)$. Although these validations were successful, it is still highly desirable to test the quality of the predictions over a larger variety of situations, regarding both the trophic state of the waters and the illumination conditions. In the present study, we will specifically concentrate on two cruises, in the South Pacific and Mediterranean Sea, and several short cruises near Hawaii, which together offer such a variety of situations.

During the BIOSOPE cruise in the South Pacific, extremely clear oligotrophic waters $\left([\mathrm{Chl}]<0.03 \mathrm{mg} \mathrm{m}^{-3}\right)$, as well as moderately eutrophic waters inside the Chilean upwelling zone $\left([\mathrm{Chl}]>1.4 \mathrm{mg} \mathrm{m}^{-3}\right)$ were encountered. The AOPEX cruise took place in the Mediterranean, and while the water types were not as varied $([\mathrm{Chl}] \sim 0.07$ to $0.15 \mathrm{mg} \mathrm{m}^{-3}$ ), we had the opportunity to sample the radiance distribution for a variety of solar zenith angles. In addition to these two cruises, we have an extensive set of radiance distribution measurements in clear waters around Hawaii ([Chl] approximately $0.1 \mathrm{mg} \mathrm{m}^{-3}$ ). All of the radiance distribution measurements were done with the camera just below the surface (at approximately $0.75 \mathrm{~m}$ ).

\section{Data set description}

The distribution of the data set is best described by Fig. 1 that illustrates the range of [Chl] and solar zenith angle for the radiance distribution data presented in this paper. The Hawaii data set has points in the [Chl] range below $0.2 \mathrm{mg} \mathrm{m}^{-3}$, but has a large range of solar zenith angles, from almost $0^{\circ}$ to over $70^{\circ}$. The AOPEX data is over a similar [Chl] range $\left(<0.2 \mathrm{mg} \mathrm{m}^{-3}\right)$ but a slightly more limited solar zenith angle range $\left(20^{\circ}<\theta_{o}<75^{\circ}\right)$. The BIOSOPE data from the South Pacific has the wider [Chl] range, from $<0.05$ to $>1.4 \mathrm{mg} \mathrm{m}^{-3}$, and $\theta_{o}$ from $10^{\circ}$ to $60^{\circ}$. The entire data set, however, does have a large hole in the 0.4 to $1.0 \mathrm{mg} \mathrm{m}^{-3}$ range.

For the AOPEX and BIOSOPE cruises, the chlorophyll concentration was determined via High-Performance Liquid Chromatography (HPLC), according to a slightly modified version (see Ras et al., 2007) of the method initiated by Van Heukelen and Thomas (2001). The simplified notation [Chl] actually represents the sum of the concentrations of the following suite of pigments: chlorophyll a, divinyl chlorophyll a, chlorophyllid a, and chlorophyll a allomers and epimers.

The upward radiance data set was obtained using the NuRADS camera system, that has been described in detail previously (Voss and Chapin, 2005). The important details are that it has an automatic filter changer, with positions for 6 spectral filters, a cooled CCD camera, and a fisheye lens to capture the complete upwelling hemisphere of data. In this paper we will use only 4 of these filters: 412, 436, 486, $526 \mathrm{~nm}$. The other 2 filters are at longer wavelengths, and there is appreciable instrument self-shadowing even in clear water. In the configuration used in most of these cruises, floatation is attached to the back of the camera, and the instrument can be floated away from the ship at a distance exceeding $50 \mathrm{~m}$, to avoid ship shadow, and tethered to the ship using a neutrally buoyant cable which combines power and communication. A portion of the data set was obtained with this system, while another portion was obtained while the instrument was in the configuration shown in Fig. 2. The system can automatically cycle through the spectral filters and collect data that are stored on the internal hard drive. It takes approximately 2 min to obtain a complete set of spectral data, and the instrument typically is set to continually cycle and collect multiple sets of radiance distribution data at each wavelength. 


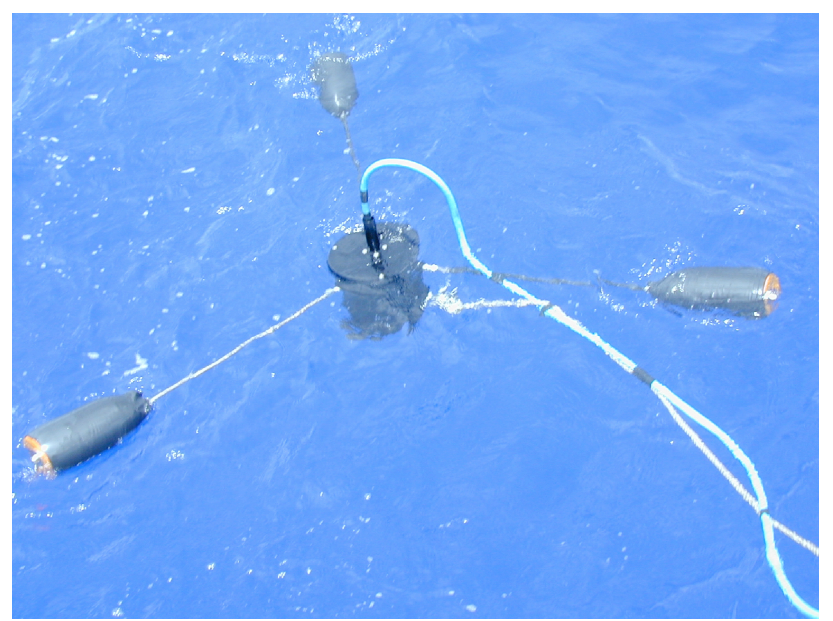

Fig. 2. Illustrating NuRads in its deployment configuration. The instrument can be floated away from the ship, suspended on the floats just below the surface.

\section{Data reduction}

Pre- and post-calibrations were done on the instruments for each cruise. The overall process to calibrate these systems is described in Voss and Zibordi (1989). An additional calibration step has been found to be required which accounts for the immersion of the dome window used in the system and its affect on the throughput of the optical system (immersion factor). This calibration step is briefly described in Voss and Chapin (2005). Images from the system have the calibration applied and result in a fisheye projection image of the upwelling radiance distribution. An example image is shown in Fig. 3.

The NuRADS instrument obtains a complete spectral set of data every $2 \mathrm{~min}$. However, since the whole upwelling radiance distribution is obtained very quickly (less than $1 \mathrm{~s}$ ), individual images often have strong features, such as wave focusing (seen in Fig. 3), which need to be averaged out. During the data reduction process we look at each image and determine, manually, where the anti-solar point is located. This point is obvious as it is the point where the wave focused light rays converge. Once this point has been determined, a computer program determines where the nadir point should be. This process uses the known angular distance between the anti-solar point and nadir, and looks for symmetry around the principal plane (plane containing the nadir and anti-solar point). When the geometry (nadir and anti-solar point in each image) has been determined, we average images taken within $10 \mathrm{~min}$. Since the image should be symmetric around the principal plane, we average each half of the image together. For the data presented in this paper, we exclude data for which only one image (both halves) are averaged, so each data point effectively represents the average of between 4 and 10 separate image halves. Images are

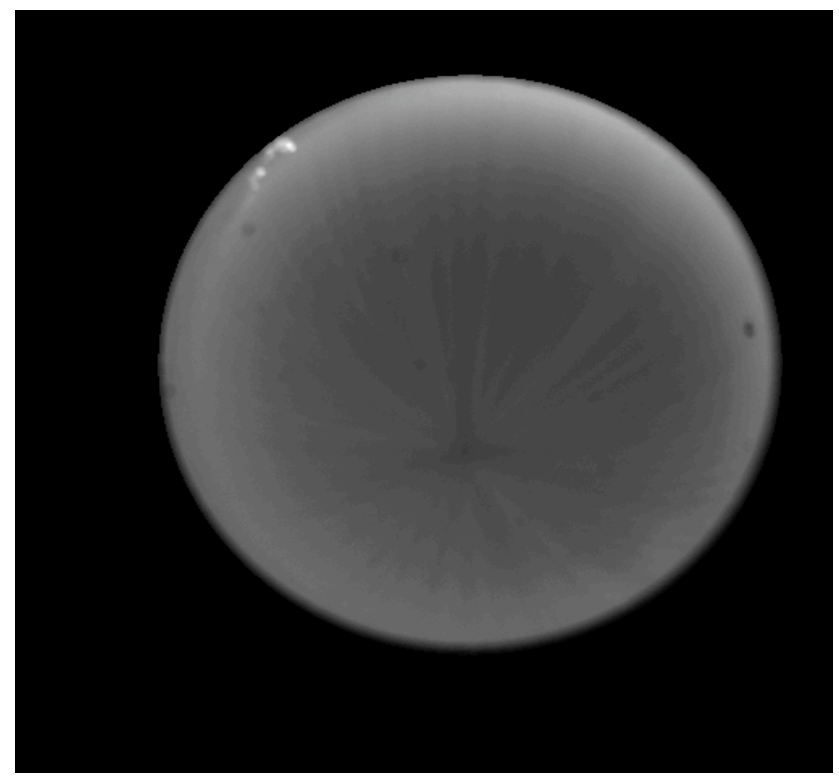

Fig. 3. Example upwelling radiance distribution image. This example is from the AOPEX cruise, on 11/8/2004. Solar zenith angle is $35^{\circ}$, [Chl $]=0.1 \mathrm{mg} \mathrm{m}^{-3}$, wavelength is $526 \mathrm{~nm}$. The geometry of this image is such that the nadir point is the center of the circle, the projection places nadir angle as directly related to the radius from the center. The edge of the circle is a nadir angle of $90^{\circ}$. The antisolar point is visible in the lower portion of the circle, defined as where the sun rays (the star pattern) converge. The azimuthal direction towards the sun is at the top of the image. The dark circles in the image are artifacts of the optical system and are masked out during the averaging process, while the bright part at the top left is probably a small portion of cable.

excluded if there is contamination in anyway (cables floating into view for example) or the anti-solar point can not be determined (most often because of clouds). In addition, to further reduce noise, each data point from each image is an average of a $3 \times 3$ pixel area (each pixel represents an angular change of approximately 0.4 degrees). An example after this data processing, for the image set containing the image shown in Fig. 3, is shown in Fig. 4.

It is important to understand that there is still environmental noise left in these images. A portion of this noise may be from our instrument system, however this is dominated by effects due to wave focusing, small scale inhomogenities in the water column, and other natural sources. To illustrate how large these variations can be we collect the $\sigma$ of each data point averaged, i.e.

$\sigma=\sqrt{\sum(x-\bar{x})^{2} / N} / \bar{x}$,

where $x$ is a specific pixel value in radiance units (not normalized), and $\bar{x}$ is the average radiance for that view geometry. $N$ is the number of pixels averaged, which is 9 pixels/image half times the number of image halves, thus between 36 and 90 pixels. Figure 5 shows $\sigma$ of the average 


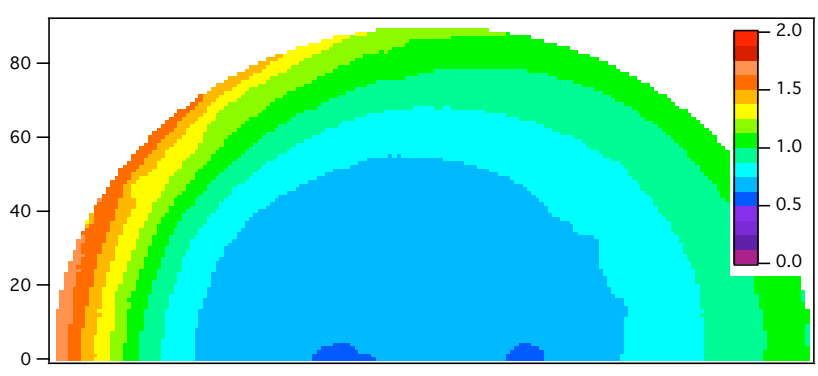

Fig. 4. AOPEX data set, solar zenith angle approximately $35^{\circ}$, data shown in $\mu \mathrm{W} \mathrm{cm} \mathrm{sr}^{-1} \mathrm{~nm}^{-1}$. This is an average of 4 images, so each point represents 8 realizations of the radiance distribution. Here $L_{\text {nadir }}$ is $0.64 \mu \mathrm{W} \mathrm{cm}{ }^{-2} \mathrm{sr}^{-1} \mathrm{~nm}^{-1}, Q_{n}$ is $3.72 \mathrm{sr}$ and $\mu_{u}$, the upwelling average cosine, is 0.44 . One can see that the wave focusing has been averaged out through this process. In this projection the anti-solar point is towards the right of the image along the $\mathrm{x}$ axis. The direction towards the sun is on the left side of the image. Nadir is represented along the $\mathrm{x}$-axis in the center of the semicircle. The nadir angle is proportional to the radial distance from the center; data exists out to 90 degrees, or the horizon.

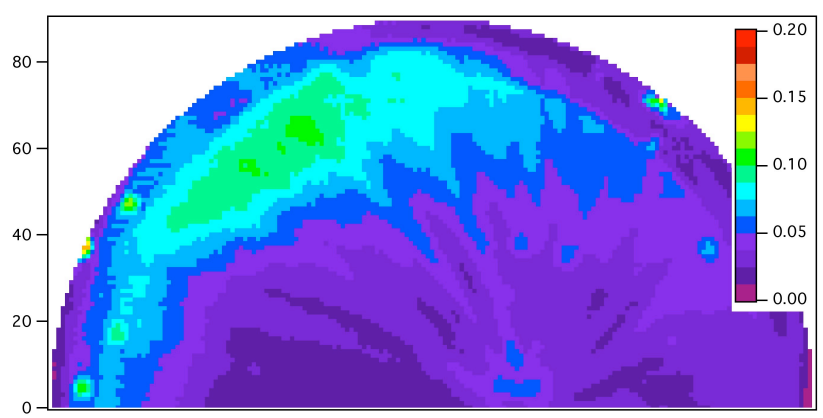

Fig. 5. $\sigma$ for each data point, in the same projection as Fig. 4. As can be seen $\sigma$ can range $>0.1$ in some areas.

shown in Fig. 4. As can be seen $\sigma$ shows the effect of wave focusing directly through the apparent sun-rays in this image. However the area with the largest $\sigma$ is towards the horizon. Figure 6 shows a histogram of $\sigma$ for one of these averages. As can be seen the peak in the histogram is on the order of 0.03 , which limits how well the data could agree with even the best model for individual points.

Any instrument, placed in the water, will have systematic measurement errors due to instrument self-shadowing. For this paper we estimated the effect of self-shadowing following a variation of the algorithm of Gordon and Ding (1992). This algorithm assumes that the shadowing is only proportional to the absorption coefficient of the water and the viewing pathlength that is directly shadowed by the instrument. The original Gordon and Ding (1992) algorithm had a simple disk casting the shadow, we extended this to a three dimensional object, with the dimensions of NuRADS, and calculated the shadowlength taking into account the refracted so-

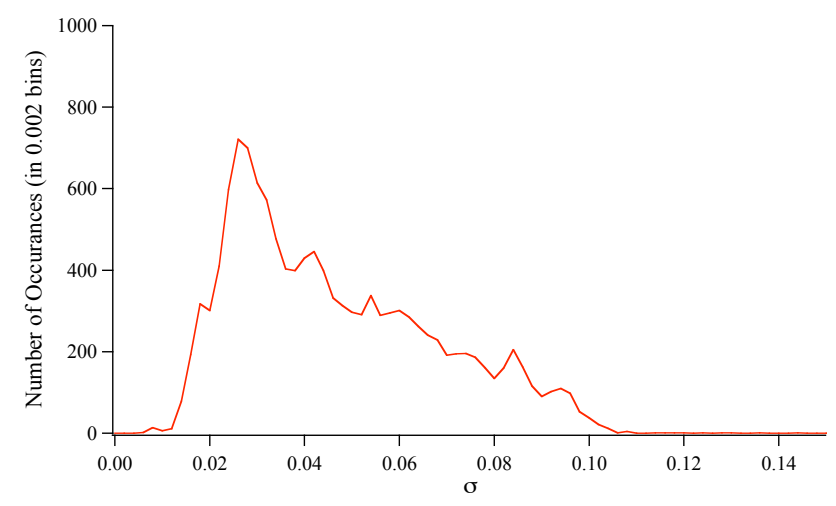

Fig. 6. Histogram of $\sigma$ of the individual data points used in above comparison with the model.

lar zenith angle, view angle, and absorption coefficient. The absorption coefficient was derived from the measured [Chl] using the model of Morel and Maritorena (2001). Because this model is a simple approximation, we only used data for which the shadowing correction was less than 5\%. It is possible to derive a more complete correction for shadowing (e.g. Helliwell et al. (1990) and Leathers et al. (2001)), however for radiance distribution measurements, such as these, complete knowledge of the seawater volume scattering function (VSF) is required. While we could use the VSF used in the model, this would influence the independence of our comparison with the data.

Our comparison will be between $L_{\text {view }} / L_{\text {nadir }}$ for the data and those predicted by the Morel et al. (2002) model and associated tables. According to Eq. (1), the ratio of a slant upward radiance to the nadir radiance is the inverse ratio of the corresponding $Q$-quantities, so

$L_{u}\left(\theta_{o}, \theta_{v}, \phi_{v}\right) / L_{u}\left(\theta_{o}, \theta_{v=0}, \phi_{v=0}\right)=Q_{n} / Q\left(\theta_{o}, \theta_{v}, \phi_{v}\right)$

here the quantity $Q_{n}$ represents the particular value for the nadir direction, i.e., $Q\left(\theta_{o}, \theta_{v=0}, \phi_{v=0}\right)$, which still depends on the sun position.

We use the quasi-contemporaneous [Chl] determinations, together with the specific illumination geometry, as determined by the instant of measurement, to enter into the $Q$ tables.

\section{Results}

Typical results are shown in Fig. 7 for one day during the BIOSOPE cruise (Station 17, 1/12/2004). $L_{\text {view }} / L_{\text {nadir }}$ for the data and model are displayed, and each data point represents a different direction or data set in that day. The resolution of direction is every 5 degrees in nadir and 15 degrees in Azimuth, thus there can be approximately 8 (5-40 degrees in nadir angle $) \times 12(0-180$ degrees in azimuth angle $)=96$ points from each radiance distribution data set. Note that the 

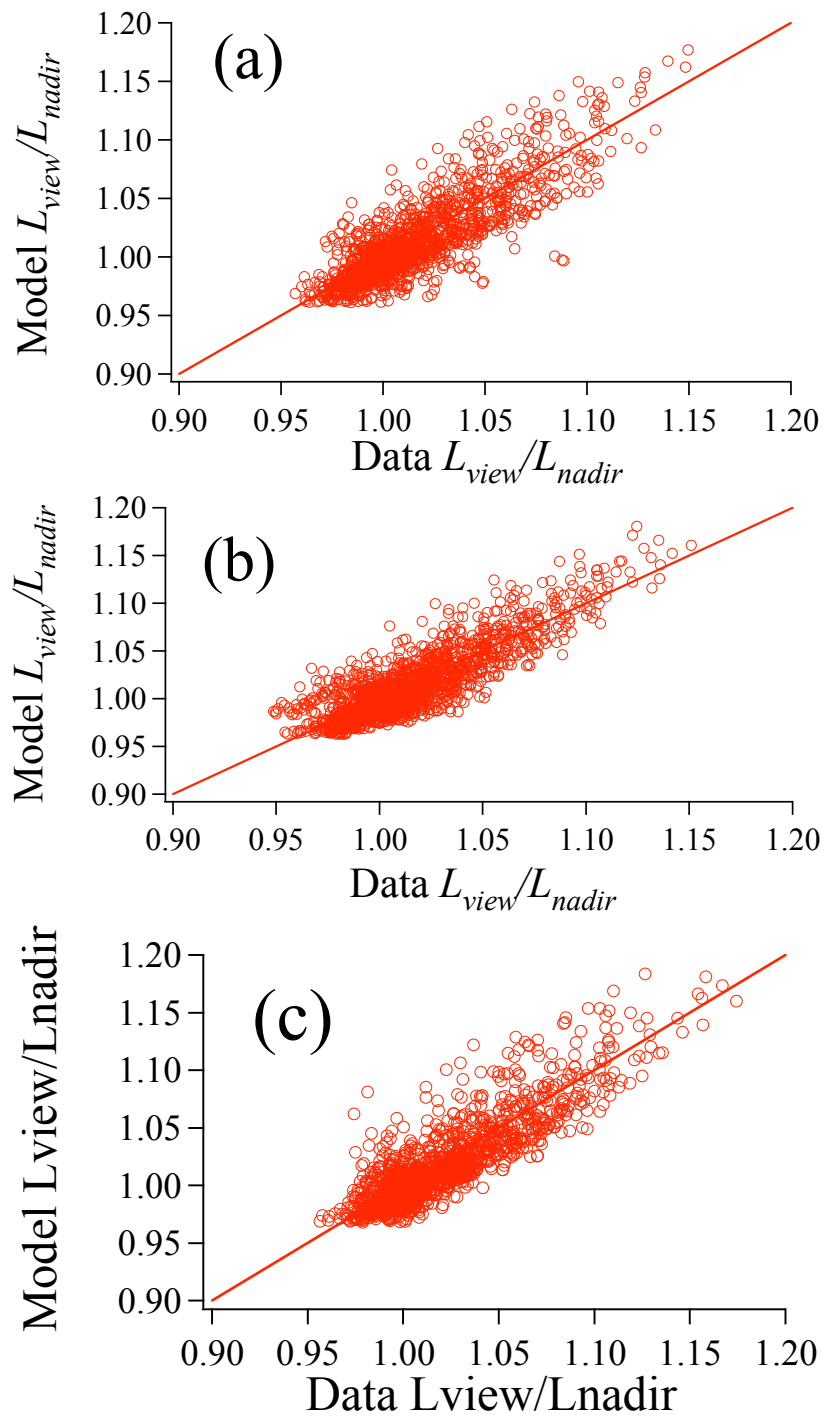

Fig. 7. Graph of model vs data of the ratio $L_{\text {view }} / L_{\text {nadir }}$ for the BIOSOPE Station \# 17; [Chl] was $0.11 \mathrm{mg} \mathrm{m}^{-3}$ in the upper layer. (a) $412 \mathrm{~nm}$, (b) $436 \mathrm{~nm}$, and (c) $486 \mathrm{~nm}$. The 1:1 line is also shown.

nadir angle is limited to upward radiances inside the Snell cone, able to emerge from the sea after refraction. For each day we calculated the deviation between the model and data in two ways, first the average difference was determined by:

Difference $=\sum($ data - model $) / N$,

and the RMS was determined by:

$\mathrm{RMS}=\sqrt{\sum(\text { data }- \text { model })^{2} / N}$.

Through our data set we can look at these factors as a function of [Chl] and solar zenith angle to see if there are any biases in the model. Figures $8 \mathrm{a}-\mathrm{d}$ shows this for the 4 wavelengths. In these figures the red dots correspond to the average difference (Eq. 5) while the bars correspond to $1 \mathrm{std}$
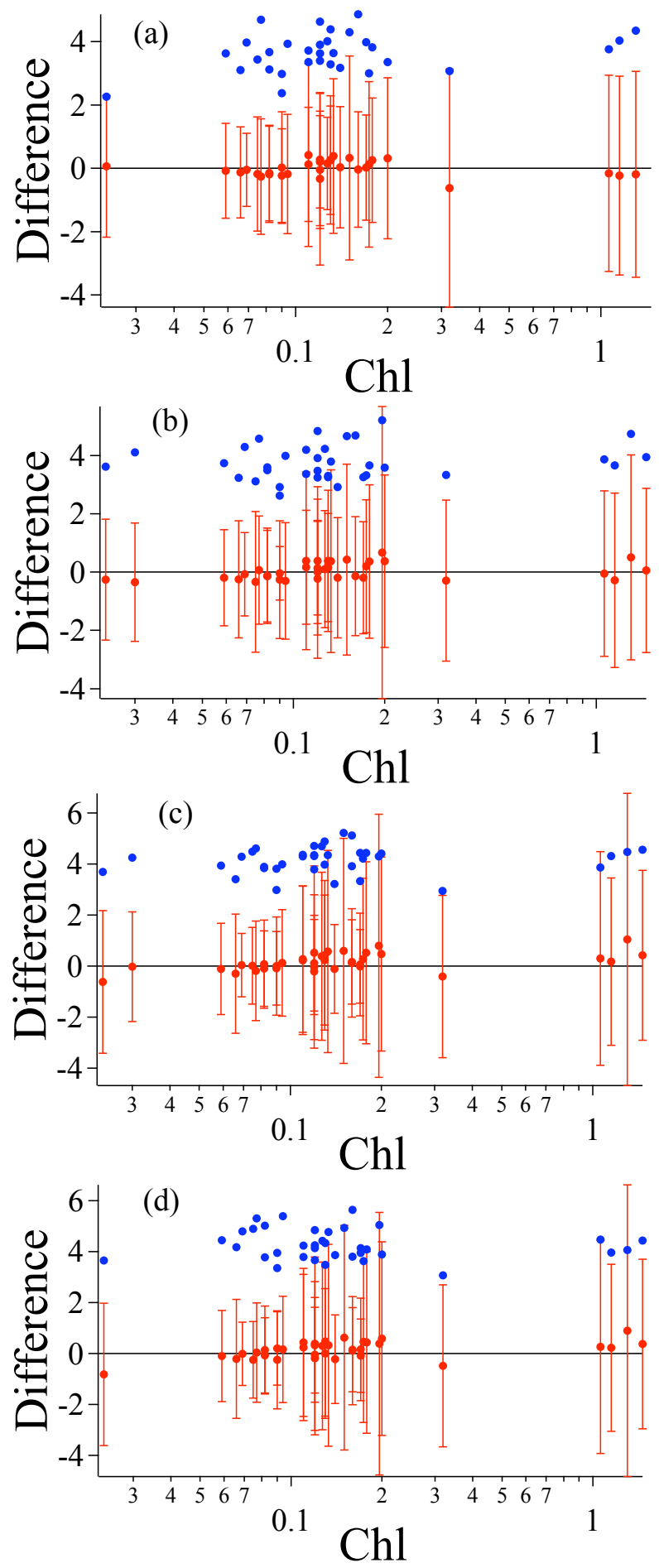

Fig. 8. Comparison for the entire data set between $L_{\text {view }} / L_{\text {nadir }}$ the model and the data. (a) $412 \mathrm{~nm}$, (b) $436 \mathrm{~nm}$, (c) $486 \mathrm{~nm}$, and (d) $526 \mathrm{~nm}$. The red dots are the average difference in agreement between the model and the data. The bars on each data point are $\pm 1 \mathrm{std}$. The blue dots are $\sigma$ obtained in the image averaging process and represents the environmental noise in the images. 


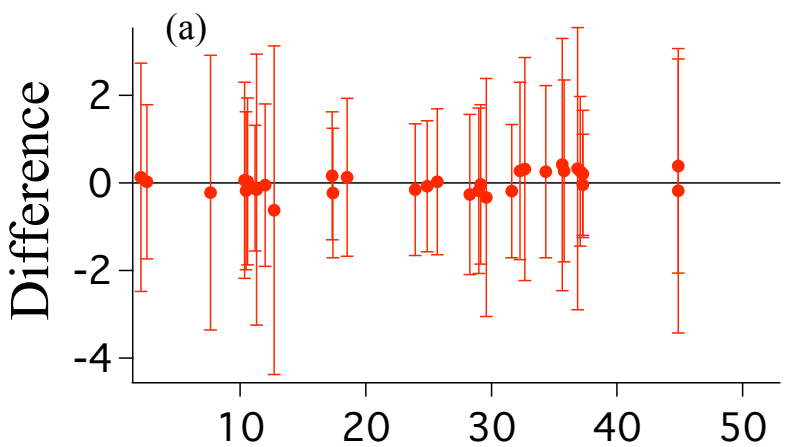

Minimum Zenith Angle

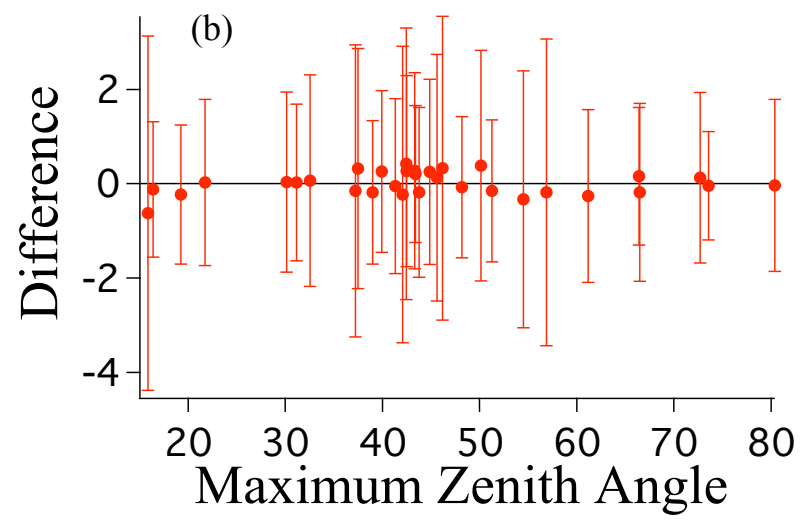

Fig. 9. Difference between the model and data $L_{\text {view }} / L_{\text {nadir }}$, shown as a function of solar zenith angle. Average difference is shown as red dots, bars are \pm 1 standard deviation. (a) Maximum solar zenith angle during that data collection, (b) Minimum solar zenith angle during that data collection. As can be seen there is no systematic trend in this difference as a function of solar zenith angle. This example shows the data at $412 \mathrm{~nm}$; there is no significant trend at any of the other wavelengths.

(Eq. 6). While collecting the difference and RMS, we also find the average $\sigma$ for the data (effectively the average of Fig. 5, for each data point). This gives a measure of the environmental noise for that point, and is shown as the blue squares.

We can also look to see if there is any bias with respect to solar zenith angle. For each day of data we collected the minimum and maximum solar zenith angle, during the data acquisition. These results are shown in Fig. 9. Figures 8 and 9 , taken together, show that in this data set there is no systematic difference with either [Chl] or solar zenith angle. In both cases the average difference is much less than 0.01 . This is well within both the standard deviation of the difference between the model and measurement and the $\sigma$ in the measurement alone. In general the RMS difference between the model and data ranged from 0.01 to 0.04 , but was mostly on the order of $0.02-0.03$. The largest difference tends to be towards the longer wavelengths where increased shadowing
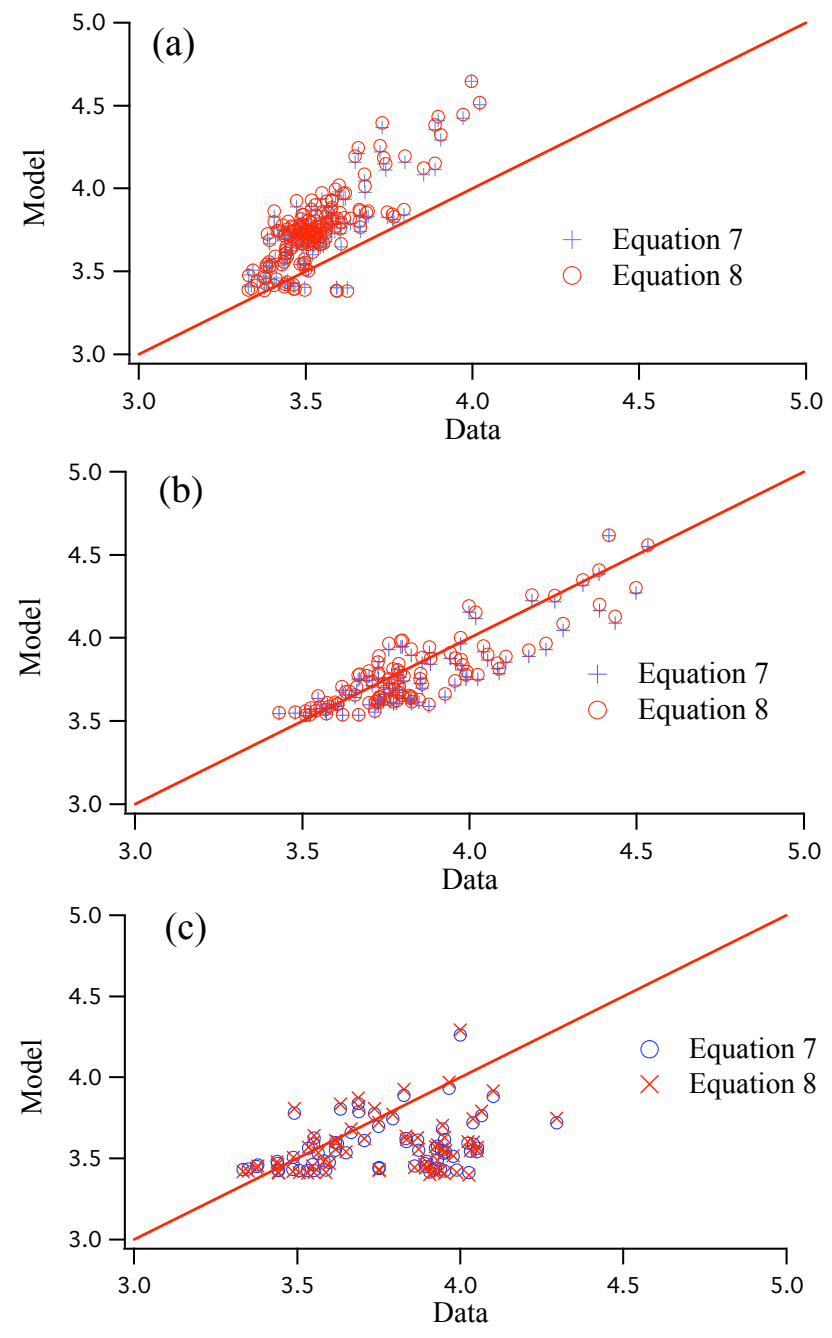

Fig. 10. Aas and Hojerslev (1999) $Q_{\text {nadir (sr) fit to data set }}$ $(486 \mathrm{~nm})$. (a) Hawaii data, (b) AOPEX (Mediterranean), (c) BIOSOPE (South Pacific). As can be seen, the best fit is with the AOPEX data. The 1:1 line is also shown.

may be causing problems in the data.

We can also use this data set to investigate the available models of $Q_{\text {nadir }}=Q\left(\theta_{o}, \theta_{v}=0, \phi_{v}=0\right)$. Aas and Hojerslev (1999), using a parameterization based on a dataset from relatively clear Mediterranean waters, predicted that $Q_{\text {nadir }}$, at blue (465-475 nm) wavelengths, should follow either:

$Q_{\text {nadir }}=5.33 \exp \left(-0.45 \cos \left(\theta_{o}\right) \mathrm{sr}\right.$, or

$Q_{\text {nadir }}=5.20-1.82 \cos \left(\theta_{o}\right)$ sr.

Morel et al. (2002) provided an alternate model, which includes a parameter for varying water types given by:

$Q_{\text {nadir }}\left(\theta_{o}, \lambda,[\mathrm{Chl}]\right)=Q_{o}(0, \lambda,[\mathrm{Chl}])+S_{Q_{n}}(\lambda,[\mathrm{Chl}])\left[1-\cos \left(\theta_{o}\right)\right]$ 

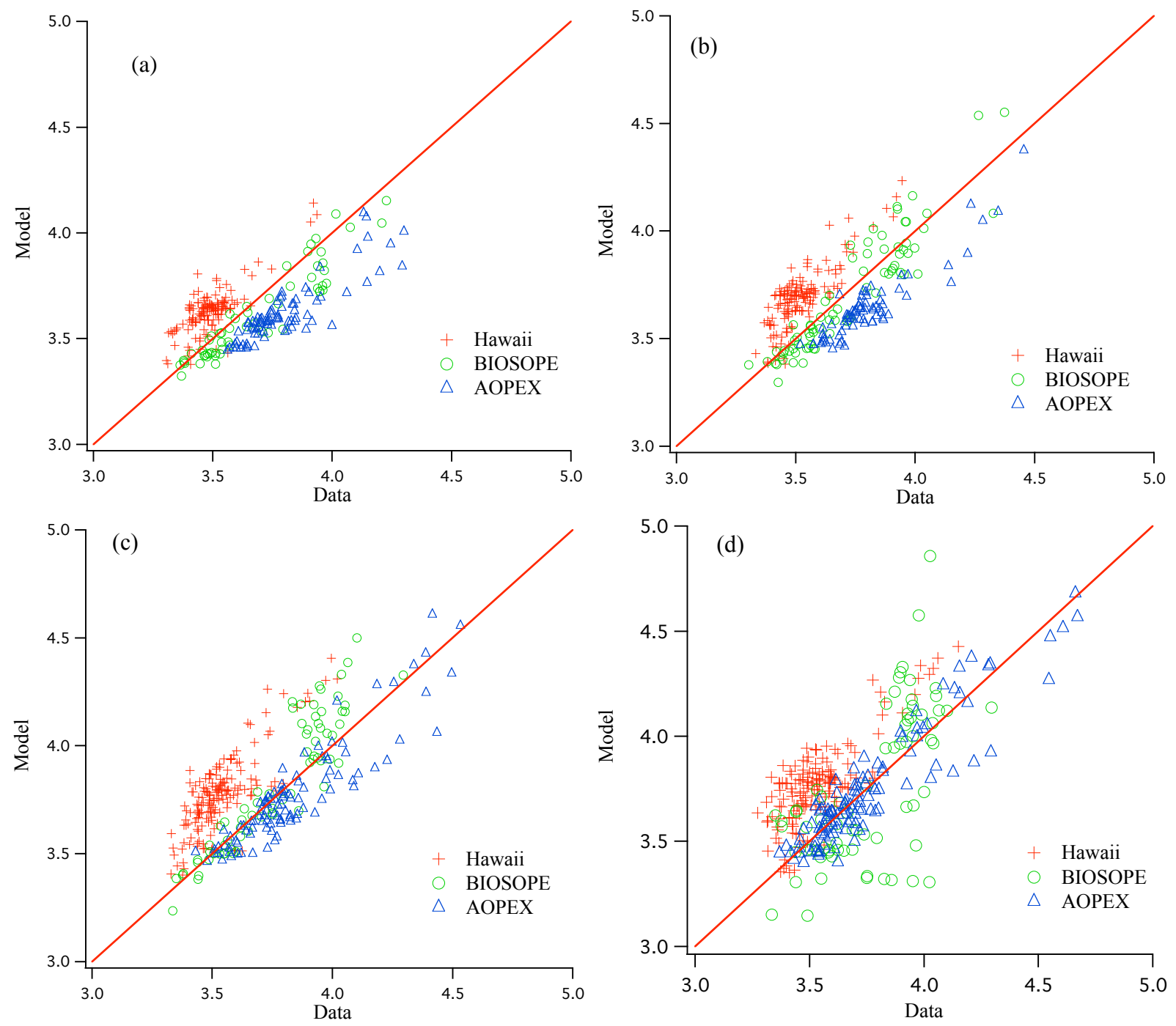

Fig. 11. Comparison of Morel et al. (2002) $Q_{n}$ (sr) model with data set. (a) $412 \mathrm{~nm}$, (b) $436 \mathrm{~nm}$, (c) $486 \mathrm{~nm}$, and (d) $526 \mathrm{~nm}$. The $1: 1$ line is also shown.

where $Q_{o}(0, \lambda,[\mathrm{Chl}])$ and $S_{Q n}(\lambda,[\mathrm{Chl}])$ can be interpolated from Table 2 in Morel et al. (2002).

The variations in both the initial term, $Q_{0}$, and the slope, $S_{Q n}$, with the chlorophyll concentration (used as bio-optical index) are by far not negligible, and are important when comparing predictions to actual data obtained in Case 1 waters with various trophic levels.

Figure 10 illustrates how well Eqs. (7) and (8) fit this data set. As can be seen, the best agreement is for the AOPEX data set, which is not surprising as this data set was obtained in the Western Mediterranean Sea, which was where the empirical factors in Eqs. (7) and (8) were determined, probably with similar [Chl] values. To quantitatively characterize this fit we calculate the RMS difference between the model and data. The RMS for these fits are $0.24 \mathrm{sr}$ (Hawaii), $0.14 \mathrm{sr}$
(AOPEX), $0.30 \mathrm{sr}$ (BIOSOPE), and $0.24 \mathrm{sr}$ for the combined data set. This also shows that Eqs. (7) and (8) are very good at fitting the AOPEX data set, and not as good at the other sites. It is obvious that a line fitted between the model and data would be significantly different than 1 for both the Biosope and Hawaii data.

Figure 11 illustrates the fit of Eq. (9) to the data set for the 4 wavelengths. The RMS differences are shown in Table 1. The model fits the data a little better than the earlier model, but there are differences in the individual data sets. Interestingly, for most wavelengths, there is a good fit of the model to the BIOSOPE data, that extend over a rather wide range of $Q_{\text {nadir values, in correspondence with the wider [Chl] range }}$ encountered during this campaign. However, we did not have [Chl] values approaching $10 \mathrm{mg} \mathrm{m}^{-3}$, thus did not see the 
Table 1. RMS difference between $Q_{n}$ (sr) model (Morel et al., 2002) and data set.

\begin{tabular}{lcccc}
\hline & \multicolumn{4}{c}{ Wavelength } \\
\hline Cruise & 412 & 436 & 486 & 526 \\
BIOSOPE & 0.11 & 0.10 & 0.14 & 0.17 \\
AOPEX & 0.20 & 0.18 & 0.12 & 0.11 \\
Hawaii & 0.16 & 0.19 & 0.26 & 0.26 \\
Combined & 0.16 & 0.17 & 0.21 & 0.20 \\
\hline
\end{tabular}

predicted maximal $Q_{\text {nadir }}$ values (close to 5 steradians) as observed in the Gulf of California (Voss and Morel, 2005).

Zibordi and Berthon (2001) have described an additional model for $Q_{\text {nadir }}$ based on data obtained in the Adriatic Sea, however this water type was significantly different than our data base, hence did not agree very well with our data and is not shown.

\section{Conclusions}

The comparison between model predictions and field data has been carried out over a wide range of environmental conditions with respect to the trophic state of the water and the sun position in clear skies. The bidirectional variation (polar and azimuthal angles) of the upward radiance distribution compared to the radiance from nadir direction, as well as the variation of this particular radiance with the sun angle have been successfully tested. The model (Morel et al., 2002) proves to be a very good tool in reproducing the various radiance distributions that we observed in our extensive data set for Case I waters. However each real, measured, radiance distribution has many features in it due to wave focusing and downwelling illumination variations. As such, while the model is able to accurately predict the average, it will never exactly fit a measured radiance distribution (nor should it be expected to do this). Much more work needs to be done to move this Case I model into the Case II regime. We currently are looking at this Case II situation with 2 data sets collected in the Chesapeake Bay, and are also looking into issues of the polarization of the upwelling light field.

As a practical conclusion, it can be added that the bidirectional corrections based on the lookup tables generated from the model, and presently applied to ocean color imagery, is sound and amply validated for Case I waters, i.e., for most parts of the global ocean. The application of such a correction is needed for a meaningful comparison of the normalized water-leaving radiances inside and between various scenes, as well as for a merging of products derived from various sensors.
Acknowledgements. This work was supported by NASA (\#NNG04HZ21C to KV). N. Souaidia and A. Chapin were, respectively, instrumental to collecting the BIOSOPE and AOPEX radiance distribution data; J. Ras who made the pigment analyses for both cruises is warmly thanked. The Hawaii data was collected in collaboration with D. Clark's group at NOAA/NESDIS, specifically M. Ondrusek and Yong Sung Kim.

The BIOSOPE and AOPEX projects and cruises were funded by CNRS and INSU (Centre National pour la Recherche Scientifique and Institut des Sciences de l'Univers), by the Centre National d'Etudes Spatiales (CNES), by the European Space Agency (ESA), and by the National Aeronautics and Space Administration (NASA), and, for BIOSOPE (a project of the LEFE-CYBER program), by the Engineering Research Council of Canada (NSERC).

The expertise of the captains, officers and crews of the research vessels Atalante (BIOSOPE) and Suroit (AOPEX) were essential for the success of these campaigns. We thank H. Claustre and A. Sciandra, the chief scientists of the BIOSOPE campaign.

This paper is dedicated to the memory of A. Chapin, a colleague and friend for over 20 years. He was instrumental in the development of the series of electro-optic radiance distribution cameras, along with many other instruments, and contributed to many experiments at sea during this long period.

Edited by: E. Boss

\section{References}

Aas, E. and Hojerslev, N. K.: Analysis of underwater radiance distribution observations: Apparent optical properties and analytical functions describing the angular radiance distributions, J. Geophys. Res., 104, 8015-8024, 1999.

Gordon, H. R. and Ding, K.: Self-shading of in-water optical measurements, Limnol. Oceanogr., 37, 491-500, 1992.

Helliwell, W. S., Sullivan, G. N., Macdonald, B., and Voss, K. J.: A finite-difference discrete-ordinate solution to the three dimensional radiative transfer equation, Transport Theor. Stat., 19, 333-356, 1990.

Leathers, R., Downes, T. V., and Mobley, C. Self-shading correction for upwelling sea-surface radiance measurements made with buoyed instruments, Opt. Express, 8, 561-570, 2001.

Loisel, H. and Morel, A.: Non-isotropy of the upward radiance field in typical coastal (Case-2) waters, Int. J. Remote Sens., 22, 275295, 2001.

Morel, A., Antoine, D., and Gentili, B.: Bidirectional reflectance of oceanic waters: Accounting for Raman emission and varying particle scattering phase function, Appl. Opt. 41, 6289-6306, 2002.

Morel, A., and Maritorena, S. Bio-optical properties of oceanic waters: a reappraisal. J. Geophys. Res., 106, 7163-7180, 2001.

Morel, A., Voss, K. J., and Gentili, B.: Bi-directional reflectance of oceanic waters: A comparison of model and experimental results, J. Geophys. Res., 100, 13 143-13 150, 1995.

Ras, J., Uitz, J., and Claustre. H.: Spatial variability of phytoplankton pigments distribution in the South Eat Pacific, Biogeosciences Discuss., accepted, 2007.

Van Heukelen, L. and Thomas, C. S.: Computer-assisted highperformance liquid chromatography method development with 
application to the isolation and analysis of phytoplankton pigments, J. Chromatogr., 910, 31-49, 2001.

Voss, K. J.: Electro-optic camera system for measurement of the underwater radiance distribution, Opt. Eng., 28, 241-247, 1989.

Voss, K. J. and Chapin, A. L.: Next generation in-water radiance distribution camera system, Proc. Soc. Photo-Optical Instrumentation Engineers, 1750, 384-387, 1992.

Voss, K. J. and Chapin, A. L.: Upwelling radiance distribution camera system, NURADS, Opt. Express, 13, 4250-4262, 2005.
Voss, K. J. and Morel, A.: Bidirectional reflectance function for oceanic waters with varying chlorophyll concentrations: Measurements versus predictions, Limnol. Oceanogr., 50, 698-705, 2005.

Voss, K. J. and Zibordi, G.: Radiometric and geometric calibration of a spectral electro-optic "fisheye" camera radiance distribution system, J. Atmos. Ocean. Techn., 6, 652-662, 1989.

Zibordi, G. and Berthon, J.-F.: Relationships between Q- factor and seawater optical properties in a coastal region, Limnol. Oceanogr., 46, 1130-1140, 2001. 